\title{
Pluralidad de significado en los bienes comunes: mirada Mapuche hacia la defensa de las aguas
}

\author{
Martín Lancaman Cárdenas \\ Universidad de Chile, Santiago, Chile \\ Email:cardenas.llancaman@gmail.com
}

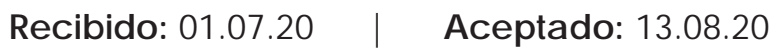

\begin{abstract}
Resumen: En lo teórico a rtíc ulo revisa la concepción de pluriversos indígenas trabaja da por A. Escobar y M. de la Cadena para a portar a la discusión sobre bienes comunes según E. Ostrom. La investigación se artic ula a partir de un caso concreto; la proyec ción de centrales hid roeléc tric a sen teritorio mapuc he y el correspondiente conflic to soc iocultural asociado. Parte importante de la investigación se centra en revisar el termino ngen (dueño del espacio), con el cual la s comunida des han a rtic ula do defensas de lossistemas de a gua sen diferentes puntos del Wallmapu. El hallazgo de este artículo es que la noción clásica de bien común debe ser critica da y abierta hacia concepciones más amplias de signific a do y que dicho paso exige además una mirada crítica de lo político.
\end{abstract}

Palabras clave: Pluriversos indígenas; giro ontológico; mapuche; bienes comunes.

\section{Plurality of meaning in the commons: Mapuc he insight towards the defense of Waters}

\begin{abstract}
The paper reviews the concept of 'ind igenous pluriverse' developed by A. Esc obar and M. De la Cadena. The goal of this research isto contribute to the debate about commons according to $\mathrm{E}$. Ostrom. The resea rch is based on a spec ific case; the projection of hydroelectric plants in Mapuche temitory and the corresponding associated socio-cultural conflict. An important part of the research is focused on reviewing the term ngen (owner of the space), with which the communities have artic ulated defenses of the water systems in different points of Wallmapu. The finding of this article is that the classic notion of the common good must be criticized and opened up to broader conceptions of meaning, and that such a step also requires a critical look at the political.
\end{abstract}

Key words: Indigenous pluriverse; ontological shift; Mapuche; common goods.

\section{Pluralidade de signific ado nos bens comuns: Mapuche olha para a defesa das águas}

Resumo: O artigo revisa o conceito de 'pluriverso indígena' desenvolvido por A. Escobare M. De la Cadena. O objetivo desta pesquisa é contribuir para o debate sobre benscomuns, de acordo com E. Ostrom. A estrutura do trabalho é baseada em um estudo de caso: projetos hidrelétric os no teritónio ma puche e conflitos ambienta is relacionados. A primeira seção desta pesquisa está focada no termo 'ngen' (proprietário do local), que tem sido usa do como uma razão para protegerbaciashidrográfic asem diferentesloca is de Wallmapu (o temitório mapuche). A visão principal deste artigo é que o conceito clássico de bens 
comuns deve ser critica do e a berto a significa dos ma is a mplos a pós o conhec imento indígena

Palavras-chave: pluriverso indígena; tumo ontológico; mapuche; bens comuns.

\section{Como citar este artículo:}

Lancaman Cárdenas, M. (2020). Pluralidad de significa do en los bienes comunes: mira da Mapuche hacia la defensa de las aguas. Polis Revista Latinoamericana, (57), 37-51. doi: http://d x.doi.org/10.32735/S0718-6568/2021-N57-1563

Neltume, Pilmaiken, Trankura y Truful Truful son cuatro zonas donde la pasada década se observaron conflictos a mbientales en temitorio mapuche con un patrón en común: la disputa y defensa de lasaguas porparte de comunidades en contra de proyectos hidroeléctricos.

Los sistemas y cuencas de agua, de los cuales depende no solo la población humana, sino también la mayoría de especiesy biodiversidad, han sido a na liza dos rec urrentemente desde el clásico Goveming the commons (Ostrom, Goveming the Commons. The evolutions of institutions for collective action, 1990), como el ejemplo paradigmático de bien de uso común. En Chile, en cambio, el ambiva lente contenido del «Código de Aguas»-que data de 1981- ha logrado que, en la práctica, el agua sea tratada como un recurso sometido a lógic as de extrac c ión y priva tiza c ión. Losa caba dos estud ios de Carl Bauer² (1998; 2004), en este sentido, han mostra do sufic ientemente que el modelo en Chile para la gestión de este bien común, «fortalece la propiedad privada y el control sobre los derechos de uso público de agua (...) y que una vez constituidos como derechos de agua, estos son gobemados porel derecho privado antes que por el derecho público»(Bauer, 2004).

Confirma ndo o antec ediendo al a ná lisis juńd ico de la priva tiza ción, la s dema ndas y esfuerzos de las comunidades mapuc he han puesto de relieve una oposición entre el uso comunita rio del teritorio y los intereses de privadossobre lasaguasal menos desde la década de 1990. En tal año, la a tención nacionale intemacional se ocupó de la proyec ción y construc ción de las hidroeléctricas Ra lco y Pangue; «el conflic to más relevante de la década de los 90, que, sin lugara dudas, trasciende al siglo XXI» (Moraga, 2001, pág. 7).

En la a ctua lidad, los pa rámetros lega les bajo los cua les se justific ó la construc ción de Ra lc o permanecen inalterados. En correspondencia, la a rtic ula ción de disc urso público por parte de comunidades que se oponen a proyectossimilares en la última década ha recogido la

\footnotetext{
${ }^{1}$ La perspectiva de Ostrom se ha vuelto indispensable primero por el uso de las categońas excluibles y rivales en consumo. En segundo lugar, por la diferenciación entre sistema de recursos y unidades de recurso y su correspondiente aplicación en el estudio de cuencas de agua y de tierras comunales (Ostrom, 2011, págs. 68; 174-224)

${ }^{2}$ Against the Current: Privatization, Water Markets, and the State in Chile (1998) -publicado por LOM en 2002 como Contra la Comiente. Privatización, mercados de agua y Estado en Chile- es un estudio interdisciplina rio con un enfoque específic o en el análisis del Código de Aguas de 1981. Un estudio comparado posterior sobre el mismo tema -de mayor amplitud temporal y con referencias al marco intemacional - fue publicado en 2004 bajo el título Siren Song. Chilean Water Law as a Model for Intemational Reform.
} 
experiencia pasada y a decua da susestrategiasy redes de colaboración. Pa ra 2017 y luego de un largo proceso de acción comunitaria y litigios judic ia les, el proyec to Central Neltume financiado por las compañías ENDESA ${ }^{3}$ - ENEL ${ }^{4}$ fue retira do definitivamente de teritorio ma puche. Las dec laraciones de J orge Weke, werken ${ }^{5}$ del Parla mento de Koz Koz resumían un año antes la perspectiva de las comunidades:

Nuestros teritorios están nuevamente amenazados por mini centra les de pasada, desparramadas como bombas de racimos, los que podrían intervenir enteramente una misma cuenca, en distintos tramos y distintos propietarios, ante eso no a dmitiremos la demolición de nuestros teritorios, por cualquier proyecto destructivo. Considerando el bien común, debemos unir las fuerzas de rechazo a las construcciones masivas de hidroelectricidad a cambio del buen vivir «Küme mogen», un concepto que para nuestros pueblos orig ina rios tiene mucho que aportar. (Weke Katrikir, 2016)

El resultado, favorable para las comunidades, se debió a que las transnacionales no pudieron solventar ec onómica mente el gasto que supuso mantener años de tramitación ante el Servic io de Evaluación Ambiental (SEA, ofic ina dependiente del Esta do) en conjunción a la fuerte a rtic ula ción social genera da. La resoluc ión exenta N¹03 de diciembre de 2015 marcaba el próximo fin de conflicto aludiendo a que la compañía de energía «desiste» de seguir con el proceso de evalua ción (SEA, 2015a), y, porende, poniendo fin a la proyección de la hidroeléc trica.

Es en este punto que creo se manifiesta un interesante problema en tomo a la disputa entre bienes comunes y los cercos privatiza dores impulsa d os por tra nsnaciona les: el proyec to -en lo formal- es desistid o por ra zones estric ta mente económic as (EL Mercurio, 2018). Sin embargo, esto no da cuenta del elemento más relevante que las comunidades adujeron persistentemente; el carácter espintual del temitorio a afectar. En efecto, parte importante del discurso público en tomo a Neltume residió en la relación del teritorio y su ngen (revisaré que se entiende porngen en el primeracápite) y en la a tención del küme mogen (buen vivir), como se puede leer en la cita anterior. Este énfasis desde la mirada mapuche es compartido en otros casos como el actual de Pilmaiken, donde se pretende preservar el «ugar sagra do Ngen Kintua nte»(Ma puexpress, 2014), o el ca so del Lof Tra nkura y su conflic to por la instalación de la Central Hidroeléc tric a Añihua raqui.

¿Qué significa este subregistro de la fuerza espiritual del temitorio que es enunciada por las comunidades mapuche? ¿Dónde puede ubicarse un elemento como este en la clásica disputa entre bienes de uso común y bienes privados? Al parecer convencionalmente en ninguno, puestanto Estado como compañías, en tanto participan del mercado, solo «movilizan informa ción y la comunican a través de los precioss (Laval \& Da rdot, 2013, pág. 145). Conocimientos indígenasque no se definan dentro de ese parámetro, entonces, no tienen representación, ni constituyen una fuente de decisión efec tiva en la resolución de conflic tos

\footnotetext{
${ }^{3}$ ENDESA (Empresa Nacional de Electricidad Sociedad Anónima) transnacional energética con sede en España.

${ }^{4}$ ENEL (Ente nazionale per l'energia elettrica) transnacional energética con sede en Italia.

${ }^{5}$ Vocero
} 
como los descritos. En otras palabras, «en el Wallmapu estos deba tes no tienen cabida en el marco de la institucionalidad y de los actores empresariales, más que como mero registro multic ultura l» (Cuadra Montoya, 2020).

Pa ra estud ia r este problema, rea liza ré en el primer a cá pite un a ná lisis so bre los doc umentos de aprobación de la Resolución Ambiental que se dio para Añihua raqui. En este punto mi principal objetivo es poner en tensión el concepto de ngen, que es fundamental, explicar algunas notas importantes de este y revisar cómo se da la mediación de este término a través de la antropología.

En segund o lugar, rec umiré centra Imente a l a utorArturo Esc oba ry su definic ión de «pluriversos indígenas» (Escobar, 2011; 2014) un proyecto de pensamiento y sentir que disputa las visiones natura liza das de economiza ción de la vida. Su colega y compañera de proyecto, Marisol de la Cadena, quien trabaja desde el presupuesto de un giro ontológico también es una referencia de peso en este texto (De la Cadena, 2015). En base a estas propuestas, a su contraste con la situación específica del conflic to en el temitorio Trankura, y a los aportes actuales sobre el pensamiento mapuche (Lanquinao \& Becerra, 2017; Melin et al, 2016) sostengo la primera de mis hipótesis, a saber, la relación entre temitorio y espinitualidad excede la noción clásica de bien común. De cómo la excede y cómo se puede abrir una plura lidad de signific a dosen elconcepto bienescomunesse tra ta portanto el segundo acápite.

Si muestro adecuadamente la hipótesis, se releva a mi juic io un segundo problema ¿Cómo comparten o se pueden articular mundos de vida diversos en el tratamiento de conflic tos a mbientales? ¿Cómo se realiza esta relación en el caso de pueblos indígenas y la conformación de un estado-nación, que se presenta de suyo como una democracia liberal fundamentada en el ideal de Modemidad? El centro de a mbas preguntas, es decir, la relación entre proyectos de sociedad divergentes, es un tema de importancia en filosofía política, mi área de especia lidad. En la sc onclusiones del artículo, dejo seña la das vía s por las cua les se puede transitar o ampliar la discusión sobre bienes comunes.

Es prec isamente la relación entre lo diverso el punto que busco a mpliaren la altemativa al desarrollo presenta da por A. Escobar. Desde mi perspectiva, la concepción de spluniversos indígenas» debe ser complementada por una exigencia crítica de justicia histórica. Si el enfoque relacional presenta do porEscobar, o la diferencia radical y equívoca (De la Cadena , 2017) pretenden imumpir transfoma doramente hacia una «ontología polític a» (Esc obar, 2014, pág. 22), entoncestal esfuerzo debe considerar que los sujetos de la polític a se construyen disocia tiva mente y en crític o «desa cuerdo»(Ra nciere, 1996).

\section{Metodología y fuentes}

El siguiente artíc ulo tiene en lo formal al menos trestipos de fuentes de lascua les se alimenta la reflexión propuesta. En tanto datos empíric os sobre cómo los conflic tos a mbientales 
han sido tra ta dos desde la instituciona lidad respec tiva se hizo uso de la pla ta forma pública del Servicio de Evaluación Ambiental, (SEA) entidad que tramita permisos ambientales y depende del Ministerio del Medio Ambiente, los protoc olos vigentes de evaluación del SEA están nomados por el reglamento $N^{\circ} 40$ del año 2013. El acceso público del sitio pemite rastrear en línea gran cantidad de documentos por caso incluyendo; informes antropológicos; correspondencia y solicitudes; resoluciones; decla raciones de impacto ambiental; actas de reunión; publicaciones oficiales. Para el uso eficiente por parte de otros investigadores se sugiere utilizar el filtro por 'comuna' para encontrar con mayor precisión el conflic to que sea de interés.

Para el seguimiento de cómo han sido recepcionados por lasagencias indígenas los diferentes conflictos ambientales, se hace uso del concepto 'discurso público', por lo que se releva $n$ en este sentid o dec la ra ciones, comunic a dosen prensa, y disc urso s rea liza dosd esde el activismo con la clara intención de disputar -tanto en la teońa como la práctica- la legitimidad de las acciones del esta do y las compañías privadas.

Una tercera fuente que permite articular metodológicamente la información disponible son recurso s textua les y public a dos que sistema tiza n o recrean los contenid os de a porta dos desde el pensamiento mapuche, se privilegian en este sentido autorías mapuche. J unto a ellasse sitúa a demásun corpus de escritos de filosofía que incluye ta nto las perspec tivas del giro decolonial latinoamericano, como en particular el giro ontológico en la versión presentada porA. Escobary M. De la Cadena.

\section{Ngen, aproximación a una disputa en tomo al significado}

El caso del proyecto Central Hidroeléctrica Añihua rra qui ${ }^{6}$ (a probada por SEA, pendiente de construc ción) a postado en el temitorio de Trankura, comuna de Curarrehue comparte caractenístic ascomunescon otroscasoscomo el del ya fracasado proyecto Centra I Neltume7, la construcción pendiente de Central hidroeléctric a Osomo ${ }^{8,}$ o la ya rechazada Central el Rincón $n^{9}$ esta última que se hubiera ubica do en el teritorio Truful Truful, comuna de Melipeuco. En espec ífic o Añihuarra qui se proyecta con una inversión de 22,00 millonesUSD, siend o controlada poraccionistas de GTD Negocios SA (Chile) y Grupo ENHOL (España).

Al igual que los anteriores ejemplos citados, la oposición al proyecto se ha articula do de manera teritorial, confluyendo para esto comunidades mapuche y otras asociaciones de

\footnotetext{
6 Tipo: Centrales generadoras de energía mayores a 3 MW. Inversión de 22,00 MM USD.

${ }^{7}$ Comuna de Panguipulli. Temitorio Neltume. Tipo: Centrales generadoras de energía mayores a 3 MW. Inversión de 781,00 MM USD. Ya desistida de evaluación en SEA.

${ }^{8}$ En el límite de las comunas Río Bueno, Puyehue y San Pablo. Teritorio Pilmaiken. Tipo: Centrales generadoras de energía mayores a 3 MW. Inversión de 75,00 MM USD. Evaluación SEA aprobada. Pemisos de construcción DGA aprobados. Pendiente de construcción

${ }_{9}$ Comuna de Melipeuco. Territorio Truful Truful. Tipo: Centrales generadoras de energía mayores a 3 MW. Inversión de 24,00 MM USD. Evaluación SEA rechazada.
} 
tipo comunitario. De manera prevalente el lof Trankura -que ha llevado la voceńa de la oposición al proyec to y la articulación de lasmovilizaciones- puso de relieve la afectación que sufrińa tanto el nguilla tuwe del lugar (a escasos 300 metros de las obras), el sistema de aguas y plantas, y el carácter sagrado de los cerros Peñewe y Punowemanke, esto último incluso reconocido en el estudio de impacto a mbiental elaborado por SEA y ta mbién en la resolución final de a probación del proyecto (SEA, 2015b, pág. 46).

Aun cuando existen razones que pudieran ca lific a rse de tipo ec onómicas pa ra la oposición al proyecto ${ }^{10}$, el corazón de los reclamos está orientado a mantener el orden y equilibrio con los ngen presentes en el temitorio; desde el punto de vista de la comunidad, la alteración y extracción de recursos hídricos en los cerros, es la intervención de todo el espacio cercano, incluyendo el eltun (cementerio), guillatuwe (espacio de ceremonia), lawen (pla ntas medic ina les). Tal c irc unstancia difícilmente es comprendida por la empresa, y mucho menos admitida. En palabras del logko Alejandro Coñuequir:

Ellos no tienen el conoc imiento. En los cerros hay un ngen, hay un ngen wigkul, hay un ngen ma wiza, hay un ngen ko, y en todos los cerros hay agua; no es mucha el agua que hay, pero existen losngen (Lof Trankura, 2016a).

Definir el término ngen es una tarea compleja. En parte, una de las traducciones usualesal castella no nos da un acercamiento suficiente, un ngen se entiende primero como el «dueño del espacio», pero a diferencia de la acepción a ntropocéntric a de «dueño»en castellano u owner en inglés, el llamado ngen es una fuerza espintual propia del lugarque está en relación tanto con plantas, minerales, humanos, hongos, animales y aguas. De ahí que en el intento de hacercomprensible esta realidad en medio de la disputa pública se recurra a la expresión «espacio sagrado»o «ugarsagrado».

Es aquí que surge un extraño problema de hemenéutica. Sobre las primeras traducciones de «dueño»y «fuerza espiritual»y también sobre la segunda interpretación de «ugar sagrado» ocurre una tercera y quizás indesea da tra ducción, la de «sitios de significa nc ia cultural». Esta última expresión a parece enunciada en el lenguaje exc lusivamente bajo la óptica de conflic tos a mbientales o socioculturales. La concreción del término es mediada a través de la antropología; de hecho en el estudio antropológico que fue admitido para la evaluación del proyecto Añihuarraqui, el especialista dedica un capítulo y un anexo a revisar tipos de «sitios de signific ancia cultura l» y su correspond iente georreferencia ción (Ba rientos Martínez, 2015, págs. 46-53; 75-96).

De ngen a «sitio» hay una distancia considerable. Un sitio, o incluso el sistema de aguas, puede medirse e identific arse en coordenadas, queda de esta manera delimita do y cercado. El ngen en cambio no puede entenderse como contenido en límites, representa en

\footnotetext{
10 i) La afectación de actividades turísticas de la zona ii) la disminución de caudal y disponibilidad de agua que afectaría los derechos de aguas de uso pemanente de miembros de la comunidad (SEA, 2015c, pág. 63); iii) la baja cantidad (8 en promedio) de empleos generados para la operación de la hidroeléctrica una vez construida la hidroeléctrica (SEA, 2015c, pág. 1)
} 
todo sentido un exceso, tema sobre el cual me extenderé unos párrafos más adelante. Ngen es in-común, si usamos el lenguaje de Marisol de la Cadena (De la Cadena, 2017), y desde mi perspectiva, la palabra primera sobre losngen esque a un cuando incluye a seres humanos, no depende de estos y a su vez tampoco lo humano se puede concebir como separado del primero. En palabras de Víctor Caniullan y Fresia Mellico:

los che somos parte del wall mapu, entendido como la tierra en que vivimos, que reúne todos los espacios materiales e inmateriales que lo componen, no solo lo que sentimos bajo nuestros pies, sino ta mbién lo que está a miba, abajo y dentro de él. En todos estos espacios existe newen, ngünen y ngen (Caniullan Coliñir \& Mellico Avendaño, 2017, pág. 44)

La necesidad teórica de preferir la expresión «sitio de signific ancia cultural»a la de ngen y la distancia hemenéutic a constatada por la transposición de interpretaciones, es operada por dos elementos complementarios; el lenguaje de la antropología y en segundo, la urgencia de la disputa ambiental. Dado que, en teoría antropológica, el lenguaje para atenderel fenómeno de lo sagrado esel lenguaje de la práctic a religiosa, el contenido de ngen ingresa bajo el código de religión y de práctica. Como práctica es algo eminentemente humano y algo que sí es susceptible de ser categorizado desde el punto de vista antropológico, entonces se lleva a cabo la vieja división entre naturaleza y cultura, organizando el «sitio» en el la do de la cultura.

¿Y qué es cultura sino acaso el fenómeno humano fundamental? (en antropología todo es cultura). De esta manera y por su énfasis en la práctica, la relación de independencia del ngen con respecto a lo humano ya no esacaso inteligible para el lenguaje. Lo cierto, desde la perspectiva mapuche, es que el ngen seguińa en el espacio, aunque no hubiese un solo ser humano para ser testigo de dicha relación o para decir una sola palabra sobre esta. Esto porque el teritorio, su ngen y su newen (fuerza) están constituidos desde antes de la presencia humana:

Como estaban recién llegando, ¿cómo iban a conocer el temitorio? Sin embargo, a través del sueño los iban guiando: 'en este lugar existe este newen', 'por aquí este newen es sumamente delicado, no se le puede contradecir, se puede enojar', 'en este lugarse van a asentar, a quí este newen es más a pa cible, es menos delic a do', les decían a través de los sueños. (Melin, Coliqueo, Curihuinca, \& Royo, 2016, pág. 26)

Lo que sucede con «sitio de signific a ncia cultural»-o con otra s c a tegońa s como sistema de agua o bienes comunes- es que termina por reducir la palabra original, sustrayéndola de sentidos complejos, pero dejando fijo un contenido a elección del observador.

El centro de mi argumento en este acápite es el siguiente. Mientrasla palabra mapuche es relacionale integra aguas, minerales, vegetación y animales, el concepto de significancia cultural puede referirse solo al sistema de aguas y a las prácticas asociadas a ella, por ejemplo, a la necesidad de uso de agua para el ngillatun. En efecto, tal fue la lectura que fina Imente preva lec ió en la evaluación del proyec to hidroeléctrico. Bastó que se aña diera el compromiso una medida simple -temporal y geográficamente a cotada- como suspen- 
derobrasu operacionespara que entoncesse diera por superada -desde el punto de vista de la empresa y el tribunal- la discusión en este punto:

El titular, en el marco de respuesta al $\mathrm{PCI}$, para liza rá las activida des construc tivas tres días antes de la ceremonia y durante la misma, de tal forma de que no exista interferencia a lguna en la ceremonia y en la calidad del agua del estero Añihuerraqui (SEA, 2015b, pág. 364).

Esta reducción de significa do escomprensible dentro de la dinámica del conflic to: la categońa «sitio de signific a ncia cultura l»y la másespecífic a definic ión de «sistema de a gua s»son criterios que sí pueden ser admitidos como argumento y ser comparables y medibles. En otras palabras, a diferencia de ngen, «sitio »sí puede llenar una casilla de medidas de mitiga ción o compensa ción (SEA, 2015b, págs. 362-364). En vista a la necesidad de intercambiar informa ción y comunic a rexperienciassobre la vivencia en el espac io, esque lascomunidades pueden aceptar la reducción del nuevo término, a un cuando es insuficiente. La mediación a través de la antropología, es la manera de haceringresar en el caso de un juic io o un estudio, los contenidos propios ma puc he, pero en categoría ajenas. De no existir esta posibilidad de registro, es dudoso incluso que interpretaciones como la de «espacio sagrado»o «fuerza espintual» hubiesen podido ser consideradas en espacios formales de disc usión como un tribunal ambiental o una comisión de evaluación.

Lo importante aquí es, que se pudiera mantener un rango más amplio de discurso, que involuc re los elementos como son presentados desde la perspectiva mapuche, con todas sus implic ancias, relaciones, polític as y ec onómic as: parte de los argumentos en contra el día que se aprobó la resolución de Añihuaraqui, dejaron una buena síntesis de este enfoque:

La afectación de un lugar sagrado de enome importancia y que involucra un complejo temitorial interc onectado y cuya protec ción se estipula en normasintema cionalescomo el Convenio 169 de la OTy la declaración de la ONU sobre derechosde los Pueblos indígenas; La transgresión de los planes de vida de referentes colectivos y tradicionales Mapuche, cuyo modelo de desarrollo es distante a estos niveles de intervencionismo, donde numerosas familias vienen proyectando el turismo, la a gro ecología y otras activida des sustentables; Se estima que estos proyectos son prácticas disc rimina torias, racista s y colonia listas, que se emplazan en teritorios anc estra les y que dañan gravemente la integrida d cultura l y esp iritual de comunida desMa puche (OLCA, 2015)

\section{Pluniversos, una lectura teónica para las diferencias}

Para superar esta brecha de interpretación sostengo que los postulados de Arturo Esc obar pueden semos de ayuda. La inic ial preoc upa ción del investiga dor por modelos altemos al desa rrollo (Escobar, 1996) ha hecho una transición gradual ha cia la a fimación de pluriversos (Esc obar, 2012). En espec ćfic o, el giro y atenc ión so bre pluriversosind ígenasma rc a una a pertura que se intenciona como «una manera de mirar la rea lidad que contrasta con la suposición del 'Mundo-Uno', de que hay una sola realidad a la cual corresponden múltiples culturas, perspec tiva so representac iones subjetivas»(Esc obar, 2014, pág. 145). 
La apertura de Escobar es especialmente pertinente para el a nálisis que propongo puesto que considera en el entra ma do una cuestión que es funda mental para entender la diferencia entre ngen y la más básica y acotada «sistema de aguas»: la exigencia de un giro ontológico descrito en términos de relación. De tal manera se genera una posibilidad más amplia hacia cambios de envergadura y de crítica a los actuales modelos de desarrollo, democracia y Estado. En palabras del autor:

estos mund os no-libera les están basa dos-en-lugar y pueden ser c a ra c teriza dos como instancias de cosmovisiones u ontologías relacionales. Ontologías relacionales son aquellas que evitan la división entre natura leza y cultura, entre individuo y comunidad, y entre nosotros y ellos que son fundamentales para la ontología modema. Esto quiere decir que algunas de las luchas en Ecuador y en Bolivia (y en otras partes del continente, incluyendo las luchas por la autonomía en Chiapas y Oaxaca, así como las luc ha s indíg enas y a fro y otras luc has ca mpesinas en Colombia, Penú, Guatemala, y otros países) pueden leerse c omo luc has ontológic as (Esc obar, 2012, pá g. 48)

Visto desde este enfoque, parte de las soluciones de E. Ostrom a las disputas sobre lo común bien podrían ser complementadas, sobre todo en el sentido de los bienes comunes a los que ella identifica como «recursos renovables» y donde las aguas son un ejemplo detallado. Las premisas de Ostrom en el caso pueden ordenarse a modo general por medio de dosgrandesgrupos; 1) los RUC (recursos de uso común) deben de mantenerse en el tiempo y 2) las comunida des loca les-a través de reg lasflexibles- son c a pa ces de ser efectivas en el resulta do de mantención y gestión de esos RUC.

Para estudiar entonces el ejemplo de las aguas, la economista acude a una delimitación precisa entre lo que es el aceno -que dado elcaso puede interpretarse como el sistema de a guas en su tota lidad- y la sunida des de recurso que, en el caso chileno corresponderían a los derechos de agua privatizados los cuales «no incluyen deberes u obligaciones hacia el interés públic o» (Bauer, 2004, pág. 55). Vale decir, que la concepción de derec hos de a gua vigente en Chile, es contradictoria con el esfuerzo de Ostrom por asegurar marcos de nomatividad comunes que contemplen el interés público o de todos quienes usan de los bienes. Con respecto a la renovación de RUC, la economista señala:

La distinción entre el recurso como aceno y la explotación de unidades de recurso como flujo resulta especia Imente útil en rela ción con los recursos renovables, donde es posible definir un ritmo de reabastecimiento. Mientras el ritmo promedio de sustracción no exceda el ritmo promedio de reabastecimiento, un recurso renovable se conserva a tra vés del tiempo (Ostrom, 2011, pág. 68)

Lo que Ostrom no puede tener en cuenta, tanto porcontexto como pordisciplina de investigación, esque la relación entre lo huma no y el sistema de aguaspuede sery de hecho es más compleja. Trankura, Truful truful, Neltume o Pilmaiken podrían mostramos que la regla de reabastecimiento no es la única que debe ser satisfecha. Ostrom, si señala acertadamente que cuando se trata de acción colectiva y de vida en tomo a un bien común como el agua, las comunidades desarrollan una «vasta nomatividad, que, de manera restringida, define el comporta miento correcto» (Ostrom, 1990, pág. 147). Ahora, si las nomas de 
tal comunidad, como es el caso mapuche, señalan la importancia de las aguas más allá de la perspectiva de recurso y consideran intrínsecamente una perspectiva rela cional que excede la acción humana, entonces la significación de bienes comunes requiere de ser ampliada, o al menos, localizada.

Para revisar la pluralidad de significados que puede tener la noción de bien común, los presupuestos de ontología relacional requieren un segundo paso; la crítica de lo político. En esto último creo, se pueden trazar interesantes claves hacia las ideas de autonomías locales y fomas de descentra lizar la democracia. En relación al paso de la ontología a la política la antropóloga De la Cadena, establece importante diferencias en su estudio de lo que ella denomina «earth beings»o «seres tierra.»

La teońa de los «serestierra » puede entenderse como la propuesta radical de que determinados espacios o montañas a las que desde otra lógica denominaríamos recurso o un poco más positivamente bien común, tra scienden la dimensión históric a y expresan su propia voluntad. Sobre estos espacios suele haber conflic to de interés de significancia que enfrentan a comunidades de losAndese inic iativas de prospec ción minera. Enmarca do en el contexto quechua de la región del Cuzco, el problema de los «seres tierra »asocia do al Ausangate -una cadena montañosa- revela el mismo problema que el de ngen: una diferencia radical de significado y una perspectiva relacional profunda ${ }^{11}$.

Al igual que el temitorio o lof mapuche donde persona y tierra comparten una compleja trama de existencia, la relac ión entre sereshumanosy su espa cio -la ca dena del Ausanga te«tiene lugar relaciona Imente, oc urre en el tiempo y en el espacio rela cional y simultáneamente, impide la separación gente y temitorio, los dos son al mismo tiempo» (De la Cadena, Risør, \& Feldman, 2018, pág. 164).

Para accedera la diferencia de signific a dos, entre lo que entiende el lenguaje instituc ional, y lo que entiende la comunidad quechua, De la Cadena da un giro a la concepción de la polític a y su relación con la historia. De tal manera, el problema fundante en la disyunción de sentidospara los «seres tierra »-y esta observación vale igual para ngen- es que mientras las comunidades pueden entender tanto el enunciado de espacio como bien común y espacio como lugar sagrado, fuerza espiritual, dueño o relación ${ }^{12}$; el lenguaje de la a utoridad y de la economía desa rollista (tanto en su fase neoliberal o dependiente del Esta -

\footnotetext{
${ }^{11}$ Incluso podría argumentarse que «seres tierra» y ngen comparten la traducción inicial de «dueño de un espacio», a unque esto requerińa un estudio más profundo. A propósito de esto la referencia en Earth Beings es el capítulo 7, «The owner of the Will»; «Nazario and his father explain that Ausangate is the highest ranking earth-being; as the most powerful, it is munayniyuq, the owner of the will, endowed with the attribute of commanding» (De la Cadena, 2015, pág. 244)

12 Dada esta imposibilidad en el caso concreto de la defensa del Ausangate, el desafío a la política modema que implicaba el ser tierra fue retirado de la discusión, y quienes proponían su presencia, rehicieron su petición recumiendo a aquello que podía ser reconocido; el medio ambiente

(De la Cadena, 2017).

${ }_{13}$ Distintos pueblos originarios cuestionan como solución suficiente las políticas post-neoliberales, que recuperaban el rol del Estado en la Economía, pero persisten en mercantilizar los temitorios con lógicas extractivas (Cuadra Montoya, Afimar los bienes comunes desde el Wallmapu, 2020)
} 
do ${ }^{13}$ ) solo puede comprender la equivalencia de bien común o la más atrasada de recurso natural.

La polític a modema es, dentro de un modo de lo posible, que requiere reconocimiento histórico. Esto signific a que en la polític a a quello que no pueda ser históricamente verificado, no es, ni sujeto ni objeto de la política, pues su estatuto es dudoso en el mejor de los casos. Este es un criterio que no necesita ser probado. El hecho de que para que se incluya algo en la polític a modema, ese algo que se incluya, tiene que ser históric 0 , ese requisito de ser históric 0 , es una condic ión, es un requisito que no necesita ser probado. Es fundamento indisputable y punto c iego, incluido, en la definición de rea lidad que hace a la polític a y que la polític a hace. Porlo tanto, desenceguecer ese punto, ofrece la posibilidad de exponer la auto evidencia sobre la que reposa el estatuto ontológico de la polític a modema (De la Cadena, 2017).

Introduc ir en la propuesta de pluriversos una noción crítica de historia y su interdependencia con el par modemidad/colonia lidad (Dussel, 1992; Mignolo, 2007; Q uija no 2007) tiene a mi juicio la ventaja de apuntaral lugar preciso donde es necesario incluir la pluralidad de significa do: la discusión polític a y pública. $O$, en palabras de J . Butler, «estudiar detenidamente el problema de la temporalidad y la política de este modo puede abrir un enfoque diferente de la diferencia cultural»(Butler, 2010, pá g. 148).

Por un camino diferente, A. Esc obar esta blece una conclusión similar; el pa so e imupción en la sforma spolític as del continente. La crítica del mundo-Uno, está orientada en el investigador colombiano a revisarla exigencia de un «mundo donde caben muc hos mundos»; en los últimos años ese tránsito desde las a ltemativas al desarrollo a los pluriversos indígenas ha llevado a considerar a Escobar especialmente los planteamientos del Buen Vivir (Sumak Kawsay):

el buen vivir debe considerarse en el contexto de toda una gama de innovaciones constitucionales pioneras, incluyendo el replanteamiento del Estado en témininos de plurinacionalidad, de la sociedad en términos de interculturalidad, una noción amplia e integral de los derechos (incluidos los derechos a la naturaleza, abajo), y un modelo de desarrollo refomado, cuyo objetivo es precisamente la realización del buen vivir. Todas estas innovaciones, además, deben ser consideradas como multic ultura les, multi-epistémic as, y en témininos de procesos de construcc ción política profundamente negocia dos y a menudo contra dictorios. Es claro, sin embargo, que el buen vivir constituye un desafío a nociones de desarrollo de larga data (Escobar, 2012, pág. 46).

\section{Conclusiones: hac ia la apertura de lo político}

La distancia entre los téminos mapuc he para determinar su relación con el teritorio y las nociones más clásicas de bien común comporta una diferencia radical de significación: mientras que el lenguaje usa do en el discurso público para realizar la defensa de lasaguas se realiza de forma relacional, en correspondencia a la experiencia mapuche del teritorio, en el caso de los bienes comunes existe un horizonte de significa do más restric tivo que no da cabida a toda la compleja relación que esquerida enunciarporparte de lascomunidades. 
Un desa cuerdo de esta naturaleza, o la constata ción que diversos mundos de vida entran en conflicto en este tipo de casos, produce además que difícilmente se puedan cumplir expec tativaso palabrasy que la mediación de la a ntropología no siempre resulte efectiva, o que incluso muchas veces pueda ser instrumentaliza da. Tal como a lgunos werken declara ron en julio de 2015 cuando se había resuelto la aprobación del proyecto Central Hidroeléctrica Añihuarraqui:

No puede serposible que la afectación de un proyecto a un camino tenga la misma ponderación de losimpactos de este mismo proyec to a la vida espinitual de todo un Lof Mapuche. Y para apoyarse en su decisión los Seremis dieron los argumentos más imisorios, que Chile necesita energía, que sin energía no hay turismo, que estamos conformes con las medidas de mitiga ción, que hay que reactivar la economía o que simplemente la ley nos ampara. (Hua iquila o, 2015).

El a porte de la concepción de pluriversospuede ayudaren una comprensión másprofunda y relevante de esta divergencia: la pluriversalidad, o las «variadas o posiciones planetarias al pensamiento único» (Mignolo, 2007, pág. 33), es más que una proposición simple de diversidad a crítica; lleva apa reja da una construcc ión y exigencia de justicia históric a y una comprensión radical de la política y sus circuitos por los cua les se constituye:

el pluriverso da lugar a coherencias y c ristaliza en prácticas y estructuras a tra vés de procesos que tienen mucho que ver con los significados y el poder; de esta manera esto se puede ver en términos de una multiplicidad de mundos. Con la ontología modema, como ya se ha sugerido, ciertas construcciones y prácticas han llegado a ser prominentes. Estos supuestos inc luyen la prima cía ontológica de los seres humanos sobre los no-humanos (Escobar, 2012, pág. 47)

Con respecto a la problemática de los bienes, incluso la visión más clásica de E. Ostrom puede sercomplementada por un a nálisis local desde la mirada de pueblosindígenas y en este caso, desde la perspectiva mapuche de aproximación a losproblemas de la política y el teritorio. Incluso la economista reconoce que además del lenguaje institucional y la necesaria sostenibilidad económic a de los recursos de uso común, una visión desde las culturas y lascomunidades es necesaria pues, se ha de «tomar en cuenta a tributos específicos de los sistemas físicos relacionados, perspectivas culturales del mundo y relaciones polític as y económic as que existen en el escenario»(Ostrom, 2011, pá g. 148).

Una a fimmación más a mplia de la política es necesaria, una que rebase los contenidos de reconocimiento, y en la que puedan ser comprendidos cualitativamente los contenidos surgidos desde los pueblos. En tal sentido una práctica de autonomía desde lo local, estańa en mejor posición que directric es venidas desde a utorida desextema sque son percibidas como atenta toria s contra «el sistema de vida y costumbres, la identidad y que atenta también con nuestra espinitualidad»(Lof Trankura, 2016b).

De no avanzarse en una crítica de la política y una revisión inédita de los significados de ngen, newen u otros, el esc enario de evalua ción de proyectos seguirá siendo una muestra de multiculturalismo neoliberal extractivo (Cuadra Montoya, 2014, pág. 156) y, además, 
seguirá obedeciendo a construc ciones coloniales que se mantienen en la vertic alidad del mundo- Uno.

\section{Agradecimientos}

El a utor de este texto es Licencia do en filosofía por Universidad Alberto Hurta do y candida to a Magíster en Filosofía por Universidad de Chile. Como lugar de enunciación y para transparencia de los lectores es importante a clararque pertenezco al pueblo mapuche. Comprometido con la idea de «soberanía epistémic a» (Comunidad de Historia Mapuche, 2012), considero fundamental desde la disciplina de estudio cursada relevar los conocimientos mapuche que puedan sostener un diálogo crític o y creativo con algunas propuestas filosóficas. En interesesde investigac ión, sigo informa da mente conflic tos so c ia les, polític osy económic os que tensionan la ac tual c onforma ción del Esta do na cional y las formas democ ráticas.

\section{Referencias}

Ba mientos Martínez, M. (2015). Indaga c ión Soc ioc ultural e Identific a ción de Sitios de Significación Cultural en la Comunidad Indígena Camilo Coñoequir Loftunekul, Lof Trankura. Estudio antropológico.

Bauer, C. (2002). Contra la comiente. Privatización, mercados de agua y el Estado en Chile. Santiago, Chile: LOM.

Bauer, C. (2004). Siren Song. Chilean Water Law as a Model for Intemational Reform. Wa shington D.C., USA: Resources for the Future.

Butler, J. (2010). Política sexual, tortura y tiempo secular. En J. Butler, Marcos de Guerra (págs. 145-190). Ciudad de México, México: Paidós.

Caniullan Coliñir, V., \& Mellico Avendaño, F. (2017). Mapuche lawentuwün. Formas de medic ina mapuche. En R. Becerra , \& G. Lanquinao , Mapun kimün. Relaciones mapunche entre persona, tiempo y espacio (págs. 41-79). Santiago, Chile: Ocho Libros Editores.

Comunidad de Historia Mapuche. (2012). Ta iñ fijke xipa rakizuameluwün. Historia, colonia lismo y resistencia desde el país Mapuche. Temuco, Chile: Edic iones Comunidad de Historia Mapuche.

Cuadra Montoya, X. (2014). Nuevas estrategias de los movimientos indígenas contra el extractivismo en Chile. Revista CIDOB d'Afers Intemacionals, 141163.

Cuadra Montoya, X. (2020). Afimar los bienes comunes desde el Wallmapu. Yene(1). Recuperado de: https://yenerevista.com/2020/06/17/a fima r-losbienes-comunes-na tura les-d esd e-el-wa llma pu/ \# ftn2

De la Ca dena, M. (2015). Earth Beigns. Ecologies of practice across Andean worlds. London, UK: Duke University Press.

De la Cadena, M. (J ulio de 2017). Cuando la naturaleza no es común... o protestas desde lo incomún. Santiago, Chile: Cla se ina ugural del año aca démico 2017. Programa de Antropología, Pontific ia Universidad Ca tólica de Temuco. Recupera do de: https://www.youtube.com/watch?v=4Ine4srh8sY

De la Cadena, M., Risør, H., \& Feldman, J. (2018). Aperturas onto-epistémicas: conversaciones con Marisol de la Cadena. Antípoda. Revista de Antropología(32), 159-177. Doi: doi:https://doi.org/10.7440/a ntipoda32.2018.08

Dussel, E. (1992). 1492. El encubrimiento del otro. Hacia el origen del mito de la modemidad. La Paz, Bolivia: Plural.

EL Mercurio, I. (29 de enero de 2018). ENEL Generación abandona proyectos 
Neltume y Choshuenco. El Mercurio. Obtenido de https:// www.elmerc urio.com/Inversiones/ Notic ia s/Ac ciones/2018/01/29/Enel-G Xaband ona-proyec tos-Neltume-y-Choshuenco.aspx

Esc obar, A. (1996). La invención del tercermundo. Construc ción y deconstrucc ión del desarrollo. Caracas, Venezuela: Fundación Editorial el perro y la rana.

Escobar, A. (2012). Más allá del desa rollo: postdesarrollo y transiciones ha cia el pluniverso. Revista de Antropología Social, 23-62. doi:http://dx.doi.org/10.5209/ rev_RASO .2012.v21.40049

Esc oba r, A. (2014). Sentipensar con la tierra. Nuevas lecturas sobre desarrollo, temitorio y diferencia. Medellín, Colombia: Edic iones UNAULA.

Hua iquila o, P. (20 de julio de 2015). Análisis de werken Karilafken sobre a probación proyecto hidroeléctrico Añihuarraqui de Curarrehue. Mapuexpress. Obtenido de https://www.mapuexpress.org/2015/07/20/a nalisis-de-werkenka rila fken-so b re-a pro ba c io n-p ro ye c to-hid ro ele c tric o-a nihua rra qui-d ecurarrehue/

Laval, C., \& Dardot, P. (2013). La nueva razón del mundo. Barcelona, España: Gedisa.

Llanquinao, Gabriel, \& Becerra, Rodrigo. (2017). Mapun kimün. Relaciones mapunche entre persona, tiempo y espacio. Santiago, Chile: Ocho Libros.

Lof Trankura (Dirección). (2016a). Awkiñko. El grito de las aguas [Pelíc ula]. Obtenido de https:// www.youtube.com/watch? time_continue $=5 \& v=$ 9axg IE4Bgro\&feature =emb logo

Lof Trankura (Dirección). (2016b). Nuestra Lucha - Werken del Lof Trankura [Película]. Obtenido de https://www.youtube.com/watch?v=RD3G Gxa eykM

Mapuexpress. (20 de Septiembre de 2014). Llamado por Pilmaiken: El lugar sagrado Mapuche Ngen Kintuante se defiende. Mapuexpress. Recuperado de: https:// www.ma puexpress.org/2014/09/20/lla ma do-por-pilma iken-el-luga r-sa gra do-ma puc he-ngen-kintua nte-se-d efiende/

Melin, M., Coliqueo, P., Curihuinca, E., \& Royo, M. (2016). Azmapu. Una Aproximación al Sistema Nomativo Mapuche desde el Rakizuam y el Derecho Propio. Temitorio Mapuche: INDH.

Mignolo, W. (2007). El pensamiento decolonial: desprendimiento y apertura. En S. Castro-Gómez, \& R. Grosfoguel, El giro decolonial (pp 25-46). Bogotá, Colombia: Siglo del hombre Editores.

Moraga, J. (2001). Aguas turbias. La central Ralco en el Alto Bío Bío. Santiago, Chile: Observatorio Latinoameric ano de Conflictos Ambientales.

Mouffe, C. (1999). El retomo de lo político. Comunidad, ciudadanía, pluralismo, democracia radical. Barcelona, España: Paidós

OLCA. (15 de julio de 2015). Pese a oposición de las comunidades, Gobiemo a prueba proyecto hidroeléc tric o Añihuarraqui en Curarrehue. Observatorio Latinoamericano de Conflictos Ambientales. Recupera do de: http://olca.cl/ a rtic ulo/nota. php? id $=105491$

Ostrom, E. (1990). Goveming the Commons. The evolutions of institutions for collective action. Cambridge, UK: Cambridge University Press.

Ostrom, E. (2011). El gobiemo de los bienes comunes. La evolución de las instituciones de acción colectiva. Ciudad de México, Méxic o: UNAM-CRIM-FCE.

Quijano , A. (2007). Colonialidad del poder y clasificación social. En S. CastroGómez, \& R. Grosfoguel, El giro decolonial (pp 93-126). Bogotá, Colombia: Siglo del Hombre.

Ranciere, J. (1996). El desacuerdo. Política y filosofía. Buenos Aires, Argentina: Nueva Visión.

SEA. (29 de dic iembre de 2015a). Desistimiento que indica Resolución exenta $N^{\circ}$ 103. XIV Región de Los Ríos. Recupera do de :https://infofima.sea.gob.cl/ Doc umentosSEA / M o strarDoc umento ? d o c Id =36/75/ 1491ca e3c 7eea 38b 769080ba 7a 4755a dda 1e

SEA. (2015b). Calific a Ambienta Imente el EIA del Proyecto «Central Hidroeléctrica Añihuerraqui». SERVICIO DE EVALUACIÓN AMBIENTAL, Comisión de Evaluación. Obtenido de https://seia.sea.gob.cl/a rchivos/2015/07/22/ $\mathrm{CH}$ Anihuerraqui VF.pdf

SEA. (2015c). Estudio de Impa cto Ambiental del Proyec to «Central Hidroeléctrica Añihuerraqui». Informe Consolidado de la Evaluación, Servicio de Evalua- 
ción Ambiental, Comisión de Evalua ción Ambiental. Recuperado de: https:/ /seia.sea.gob.cl/archivos/2015/07/06/ICE CH Anihuerraqui_Semifinal.pdf

Weke Katrikir, J. (20 de Febrero de 2016). Opinión por retiro de $\mathrm{CH}$ endesa de Neltume: El triunfo ante la tiranía contra nuestros derechos humanos mapuche y no mapuche. Mapuexpress. Recuperado el 27 de junio de 2020, de https://www.mapuexp ress.o rg/2016/02/20/o pinion-p or-retiro-de-chendesa-de-neltume-el-triunfo-a nte-la-tira nia-c o ntra-nuestros-derec hos-humanos-mapuche-y-no-mapuche/ 